\title{
Variable-Voltage Class-E Power Amplifiers
}

\author{
Mustafa Acar, Anne Johan Annema and Bram Nauta \\ IC Design Chair, University of Twente, 7500 AE Enschede, The Netherlands
}

\begin{abstract}
The Class-E power amplifier is widely used due to its high efficiency, resulting from switching at zero voltage and zero slope of the switch voltage. In this paper, we extend general analytical solutions for the Class-E power amplifier to the ideal single-ended Variable-Voltage Class-E (Class-E $E_{V V}$ ) power amplifier that switches at zero slope but not necessarily at zero voltage.

The theory is verified by simulations and measurements, and show that the peak switch voltage of Class- $\mathrm{E}_{V V}$ power amplifier is lower (up to $\approx 30 \%$ ) than in the conventional Class-E power amplifier; which can be utilized to obtain e.g. higher output power (up to $\approx 200 \%$ more) with lower power-added efficiency (maximum $\approx 20 \%$ less) from technologies with low-breakdown voltages.
\end{abstract}

Index Terms - Power amplifiers, switching amplifiers.

\section{INTRODUCTION}

The Class-E power amplifier (PA) can achieve high efficiency due to its tuned load network that shapes the switch voltage to zero-voltage and zero-slope at the switch turnon moment. Many different aspects of the Class-E power amplifier (PA) have been extensively studied in the last three decades [1]- [7]. The reportedly "sub-optimum operation" with nonzero-voltage or nonzero-slope switching [3] is hardly used because of its believed inferior performance.

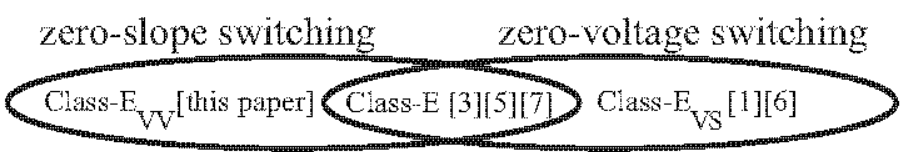

Fig. 1. Sub-optimum operation classes of Class-E PA

In [1], zero-voltage and nonzero-slope switching operation only for RF-choke Class-E PAs has been investigated and it is found that these Class-E PAs have about $10 \%$ higher tolerance to switch (transistor) output capacitance than conventional RFchoke Class-E PAs. In [6], it has been shown that zero-voltage and nonzero-slope switching (denoted as variable-slope ClassE, Class-E $E_{V S}$ (Fig.1)) operation of finite dc-feed inductance Class-E PAs allows using significantly larger switch (transistor) size (up to $110 \%$ more); which can be utilized in obtaining higher efficiency.

To the best of the authors' knowledge, analyses of nonzerovoltage and zero-slope switching Class-E PAs (denoted as Variable-Voltage Class-E, Class- $\mathrm{E}_{V V}$ (Fig.1)) have not been presented in literature. This paper presents an analysis and some discussions on Class- $\mathrm{E}_{V V}$ PAs to utilize their lower peak switch voltage feature to obtain higher output power with reasonably high power-added efficiency from transistor technologies with low-breakdown voltages. The analysis in this paper is based on closed form expressions like those presented in [5], [6] and [7] for Class-E PAs ${ }^{1}$. In the analysis, the finite dc-feed inductance $(L)$, the switch (transistor) input/output capacitance $\left(C_{\text {in }} / C_{\text {out }}\right)$ and on-resistance $\left(R_{\text {on }}\right)$ (Fig. $\left.2 \mathrm{~b}\right)$ are all taken into account. As a result of the analysis, analytical design equations are presented showing the relation between the input parameters and the circuit component values. It is shown in this paper that Class- $\mathrm{E}_{V V}$ can have up to $\approx 30 \%$ lower peak switch voltage than conventional Class-E PAs; which can be used to obtain up to $\approx 200 \%$ more output power with maximum $\approx 20 \%$ lower power-added efficiency ${ }^{2}$ assuming the same transistor size, reliable peak voltage, matching network and pre-driver.
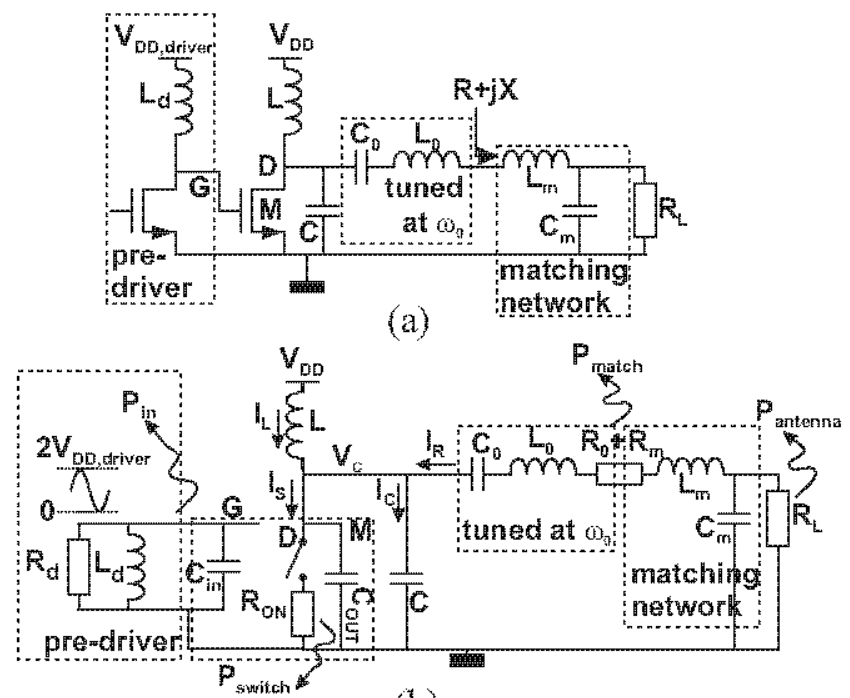

(b)
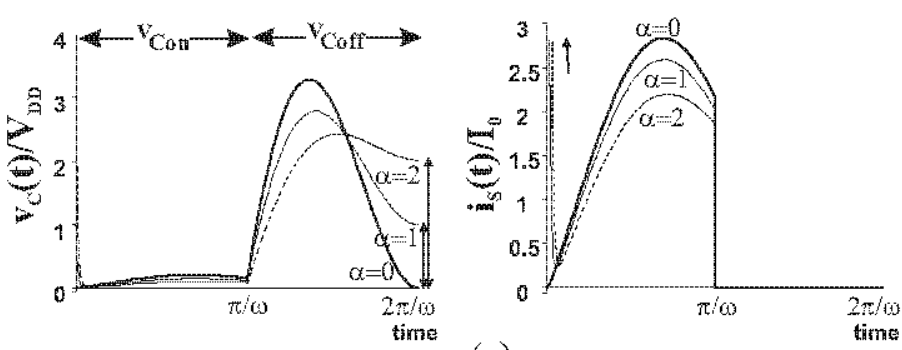

(c)

Fig. 2. (a) Class- $E_{V V}$ PA including pre-driver and matching network (b) Model of Class- $E_{V V}$ PA (c) Normalized switch voltage and switch current of Class- $\mathrm{E}_{V V}$ PA with turn-on voltage of $\alpha=2, \alpha=0$ (Class-E) and $\alpha=1$

\footnotetext{
${ }^{1}$ In [5] and [7] only Class-E operation and in [6] only Class- $E_{V S}$ operation is considered.

${ }^{2}$ Power is lost due to discharging of the capacitor $(C)$ to ground via the switch at the switch turn-on moment.
} 


\section{ANAlysis of Class-E $V V$ POWER AMPlifier}

A single ended switching PA topology and its model are given in Fig.2a and Fig.2b respectively. An analytical solution for the model in Fig. $2 \mathrm{~b}$ to operate as a Class-E PA (e.g. switching at zero-voltage and zero-slope) is given in [5] and [7] ${ }^{3}$. In [6], switching with variable slope aspect was generalized and analytical design equations for Class- $E_{V S}$ (e.g. switching at zero-voltage and variable-slope) is given. In the current paper, the variable voltage aspect is generalized and general analytical solution for Class- $\mathrm{E}_{V V}$ (e.g. switching at variablevoltage and zero-slope) is given based on the model in Fig. 2b. If the correct input parameters and circuit element values are chosen, the circuit in Fig. 2a properly operates as a Class- $\mathrm{E}_{V V}$ PA by satisfying the following conditions (1):

$$
v_{C}(2 \pi / \omega)=\alpha V_{D D} \text { and }\left.\frac{d v_{C}(t)}{d t}\right|_{t=2 \pi / \omega}=0
$$

where $\alpha V_{D D}$ is the voltage of $v_{C}(t)$ at the moment the switch is closed; for conventional Class-E operation $\alpha=0$. However, in Class- $E_{V V}$ operation $\alpha$ is a real value ${ }^{4}$ that can be selected freely and therefore gives a degree of freedom in the design of a Class- $\mathrm{E}_{V V}$ PA. A design set $K=\left\{K_{L}, K_{C}, K_{P}, K_{X}, K_{R}\right\}$ (Table 1) can be derived that relates circuit element values and operating conditions such as supply voltage, operating frequency and output power for the switching PA in Fig.2a. In this section, closed form analytical expressions for each element of the Class- $E_{V V}$ are derived; which enables infinitely many Class- $\mathrm{E}_{V V}$ realizations, to be selected by the parameters $q=1 /(\omega \sqrt{L C}), m=\omega R_{o n} C$ ( [5], [6], [7]) and $\alpha$. The design parameters $q$ and $m$ are free variables like $\alpha$ and mathematically can be assigned any positive real value $\mathrm{e}^{5}$.

Table 1: Design Set $K$ for Class- $\mathrm{E}_{V V} \mathrm{PA}^{6}$ $K_{L}=\frac{\omega L}{R}, K_{C}=\omega C R, K_{P}=\frac{P_{O U T} R}{V_{D D}^{2} R}, K_{X}=\frac{X}{R}, K_{R}=\frac{R_{\text {on }}}{R}$

This section presents the derivation of the design set $K$ for Class- $\mathrm{E}_{V V}$ PAs.

\section{A. Circuit Description and Assumptions}

The circuit model of the Class-E PA is given in Fig.2b. For the analysis and the derivations in this paper a number of assumptions are made:

- the only real power loss occurs on $R_{L}, R_{o n}, R_{d}, R_{0}$ and $R_{m}$.

- The capacitors $C_{i n}$ and $C_{\text {out }}$ are assumed to be linear.

- the switch (transistor) operates instantly with onresistance $\left(R_{o n}\right)$ and infinite off-resistance

\footnotetext{
${ }^{3}$ Note that the analysis in [5] is extended in [7] by taking into account a non-zero switch on-resistance.

${ }^{4}$ Theoretically, $\alpha<0$ is possible however, for MOS type of switches the junction diodes get forward biased when $\alpha<0$; decreasing efficiency.

${ }^{5}$ Although mathematically $q, m$ can be assigned any positive real value and $\alpha$ can be assigned any real value, as it is shown (later) that only certain ranges results in design equations that are feasible in practice.

${ }^{6} L_{0}$ and $C_{0}$ seen in Fig. 2 a can be determined from the chosen loaded quality factor $\left(Q_{L}=\omega_{0} L_{0} / R\right)$ where $\omega_{0}=1 / \sqrt{L_{0} C_{0}}$.
}

- the loaded quality factor $\left(Q_{L}\right)$ of the series resonant circuit $\left(L_{0}\right.$ and $C_{0}$ ) is high enough in order for the output current to be sinusoidal at the switching frequency

- the duty cycle is $50 \%$

Fig.2c shows the switching behavior and the switch definition used: in the time interval $0 \leq t<\pi / \omega$ the switch is closed and in $\pi / \omega \leq t<2 \pi / \omega$ it is opened. This switching repeats itself with a period of $2 \pi / \omega$.

\section{B. Circuit Analysis}

In the analysis, the current into the load, $i_{R}(t)$, is assumed to be sinusoidal. Note that theoretically this occurs only for infinite $Q_{L}$ of the series resonant network consisting of $L_{0}$ and $C_{0}$. It is however a widely used assumption [1], [3], [5] that simplifies analysis considerably: $i_{R}(t)=I_{R} \sin (\omega t+\varphi)$ In the time interval $0<t<\pi / \omega$, the switch is closed. The $\mathrm{KCL}$ at the drain node can be written as:

$$
i_{L}(t)-i_{S}(t)-i_{C}(t)+i_{R}(t)=0
$$

Relation (2) can be arranged in the form of a linear, nonhomogenous, second order differential equation

$$
\begin{array}{r}
C \frac{d^{2} v_{C_{o n}}(t)}{d t^{2}}+\frac{1}{R_{o n}} \frac{d v_{C_{o n}}}{d t}-\frac{V_{D D}-v_{C_{o n}}}{L} \\
-\omega I_{R} \cos (\omega t+\varphi)=0
\end{array}
$$

which has as solution

$$
\begin{aligned}
v_{C_{\text {on }}}= & \frac{q^{4} \sin (\omega t+\varphi) m-\left(q^{2}-q^{4}\right) \cos (\omega t+\varphi)}{1+\left(m^{2}+1\right) q^{4}-2 q^{2}} p V_{D D} \\
& +V_{D D}+e^{a \omega t} C_{o n_{2}}+e^{b \omega t} C_{o n_{1}}
\end{aligned}
$$

where, $a=\frac{-1+\sqrt{1-4 q^{2} m^{2}}}{2 m}, b=\frac{-1-\sqrt{1-4 q^{2} m^{2}}}{2 m}$ and $p=$

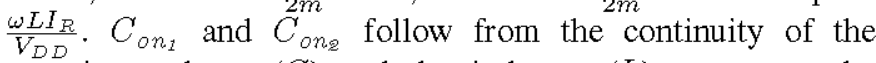
capacitor voltage $(C)$ and the inductor $(L)$ current at the switch-on moment.

In the time interval $\pi / \omega<t<2 \pi / \omega$, the switch is opened. Then, in the Class- $\mathrm{E}_{V V}$ PA the current through capacitance $C$ is

$$
i_{C}(t)=\frac{1}{L} \int_{\pi / \omega}^{t}\left(V_{D D}-v_{C_{o f f}}(t)\right) d t+i_{L}\left(\frac{\pi}{\omega}\right)+i_{R}(t)
$$

Relation (5) can be re-arranged in the form of a linear, nonhomogeneous, second-order differential equation

$$
L C \frac{d^{2} v_{C_{o f f}}(t)}{d t^{2}}+v_{C_{o f f}}(t)-V_{D D}-\omega L i_{R} \cos (\omega t+\varphi)=0
$$

which has as solution

$$
\begin{aligned}
v_{C_{o f f}}(t)= & C_{o f f_{1}} \cos (q \omega t)+C_{o f f_{2}} \sin (q \omega t)+V_{D D} \\
& -\frac{q^{2}}{1-q^{2}} p V_{D D} \cos (\omega t+\varphi)
\end{aligned}
$$

$C_{o f f_{1}}$ and $C_{o f f_{2}}$ follow from the Class- $\mathrm{E}_{V V}$ conditions (1).

It follows from (4) and (7) that $v_{C_{o n}}(t)$ and $v_{C_{o f f}}(t)$ can be expressed in terms of $V_{D D}$ and $\omega$ hence be solved analytically only if $\varphi, q, p, m$ and $\alpha$ are known. The derivation of the four parameters $\varphi, p, q, m$ and $\alpha$ is the next step in the solution. 
By using the continuity of the inductor current and the capacitor voltage at the switch turn-off moment two independent equations follow that have the same format:

$$
\begin{aligned}
f_{i}(p, q, \varphi, m, \alpha)= & p a_{i}(q, m, \alpha) \cos (\varphi)+p b_{i}(q, m, \alpha) \sin (\varphi) \\
& +c_{i}(q, m, \alpha)=0, \text { where } i=1,2
\end{aligned}
$$

The variables $p$ and $\varphi$ can be solved by using $f_{1}(p, q, \varphi, m, \alpha)$ and $f_{2}(p, q, \varphi, m, \alpha)$ in terms of $q, m$ and $\alpha$ as given in the appendix. Here, $q, m$ and $\alpha$ are free variables that can mathematically take any positive real value.

\section{Design sets for Class- $E_{V V}$ operation}

The results of the mathematical derivation of the solutions leading to Class- $\mathrm{E}_{V V}$ operation can be used to derive an easyto-use design procedure for Class- $\mathrm{E}_{V V}$ PAs. Using the result of the derivation for $p(q, m, \alpha)$ and $\varphi(q, m, \alpha)$, analytical expressions for the design set $K=\left\{K_{L}, K_{C}, K_{P}, K_{X}, K_{R}\right\}$ can readily be derived.

$\underline{K_{L}}$ : follows from the principle of power conservation:

$$
I_{R}^{2} R / 2+P_{\text {switch }}=I_{0} V_{D D}
$$

In this relation, $I_{0}$ is the average supply current:

$$
I_{0}=\frac{\omega}{2 \pi R_{o n}} \int_{0}^{\pi / \omega} v_{C_{o n}}(t) d t
$$

and $P_{\text {switch }}$ is the power spent on $R_{o n}$ :

$$
P_{\text {switch }}=\frac{\omega}{2 \pi R_{o n}} \int_{0}^{\frac{\pi}{w}} v_{C_{o n}}(t)^{2} d t
$$

Substitution of (9) and $p$ in (8) yields

$$
K_{L}(q, m, \alpha)=\frac{-\left(p V_{D D} q\right)^{2} m \pi}{\omega \int_{0}^{\frac{\pi}{w}}\left(v_{C_{o n}}(t)^{2}-V_{D D} v_{C_{o n}}(t)\right) d t}
$$

Since $p$ and $\varphi$ are all functions of $q, m$ and $\alpha, K_{L}$ is a function of only $q, m$ and $\alpha$.

$K_{C}$ : follows directly from the definition of $q$ and $K_{L}$ : $\overline{K_{C}}(q, m, \alpha)=1 /\left(q^{2} K_{L}(q, m, \alpha)\right)$

$K_{P}$ : can easily be found as a function of $q, m$ and $\alpha$ by using $\overline{I_{R}}=\sqrt{2 P_{\text {OUT }} / R}$ and the definition of $p$ :

$$
K_{P}(q, m, \alpha)=p(q, m, \alpha)^{2} /\left(2 K_{L}(q, m, \alpha)^{2}\right)
$$

$K_{X}:$ can be derived using two fundamental quadrature Fourier components of $v_{C}(t)$.

$$
\begin{gathered}
v_{R}=\int_{0}^{\frac{\pi}{\omega}} \frac{v_{C_{o n}}(t)}{\pi} \sin (\omega t+\varphi) d t+\int_{\frac{\pi}{\omega}}^{\frac{2 \pi}{\omega}} \frac{v_{C_{o f f}}(t)}{\pi} \sin (\omega t+\varphi) d t \\
v_{X}=\int_{0}^{\frac{\pi}{\omega}} \frac{v_{C_{o n}}(t)}{\pi} \cos (\omega t+\varphi) d t+\int_{\frac{\pi}{\omega}}^{\frac{2 \pi}{\omega}} \frac{v_{C_{o f f}}(t)}{\pi} \cos (\omega t+\varphi) d t \\
K_{X}(q, m, \alpha)=v_{X} / v_{R}
\end{gathered}
$$

$\frac{K_{R}}{K_{R}}$ : follows from the definition of $m$ and $K_{C}$ : $\overline{K_{R}}(q, m, \alpha)=K_{C}(q, m, \alpha) / m$

\section{Efficiency and Output Power of Class- $E_{V V}$}

Taking the three loss mechanisms ( $P_{i n}, P_{\text {switch }}, P_{\text {match }}$ ) shown in Fig. 2 and the existence of certain switch (transistor) breakdown-voltage $^{7}\left(V_{B D}=z(q, m, \alpha) \cdot V_{D D}\right)$ into account the efficiency and the power on the antenna can be expressed:

Drain Efficiency $(\eta)$ : derived as a function of $q, m$ and $\alpha$.

$$
\eta(q, m, \alpha)=1-\frac{P_{\text {switch }}}{V_{D D} I_{0}}=1-\frac{\int_{0}^{\frac{\pi}{w}} v_{C_{o n}}(t)^{2} d t}{V_{D D} \int_{0}^{\frac{\pi}{\omega}} v_{C_{o n}}(t) d t}
$$

Power-Added Efficiency $(P A E)$ : as a function of $q, m$ and $\alpha$.

$$
\begin{aligned}
& P A E(q, m, \alpha)=\frac{P_{\text {antenna }}-P_{i n}}{V_{D D} I_{0}} \\
& \quad=\eta(q, m, \alpha)\left(\frac{1}{1+\frac{\sqrt{n-1}+Q_{L}}{Q_{m}}}-\frac{c_{1} c_{2}}{2 d^{2} Q_{d}} \frac{K_{C}(q, m, \alpha)}{K_{P}(q, m, \alpha)}\right)
\end{aligned}
$$

where, $P_{\text {antenna }}=P_{\text {OUT }}-P_{\text {match }}, n=\frac{R_{L}}{R}, P_{\text {in }} \approx$ $\omega C_{\text {in }} V_{D D_{\text {driver }}}^{2} /\left(2 Q_{d}\right), c_{1}=C_{\text {in }} / C_{\text {out }}, c_{2}=C_{\text {out }} / C, d=$ $V_{D D} / V_{D D_{\text {driver }}} \cdot Q_{m}$ is the quality factor of the inductors $L_{m}$ and $L_{0} \cdot Q_{d}$ is the quality factor of $L_{d}$ (Fig. 2b).

Power on Antenna $\left(P_{\text {antenna }}\right)$ : as a function of $q, m$ and $\alpha$.

$$
P_{\text {antenna }}(q, m, \alpha)=\frac{K_{P}(q, m, \alpha)}{z(q, m, \alpha)^{2}} \frac{n}{1+\frac{\sqrt{n-1}+Q_{L}}{Q_{m}}} \frac{V_{B D}^{2}}{R_{L}}
$$

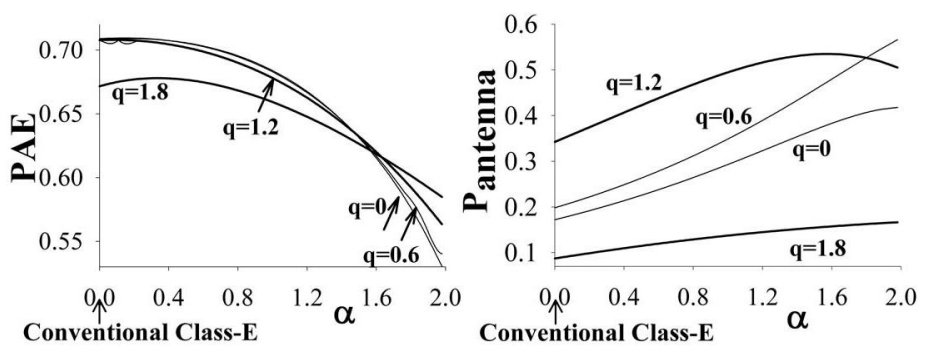

Fig. 3. $P A E$ and $P_{\text {antenna }}$ using technology and design parameters in [4]

The value of $c_{1}, d$ and $V_{B D}$ depend on the characteristics of the transistor technology. For a certain operation frequency and transistor technology, $m$ only depends on the value of $c_{2}$ since $m=\omega \beta / c_{2}$ where $\beta=R_{o n} C_{o u t}$ which depends on the transistor technology. Therefore, for a given transistor technology, impedance transformation ratio $(n), Q_{m}$ and $Q_{d}$, $P A E$ and $P_{a n t e n n a}$ both are a function of only $q, m$ and $\alpha$. In Fig. 3, $P A E$ and $P_{\text {antenna }}$ are plotted as a function of $\alpha$ for a few values of $q$ using the design and technology parameters in [4]. In [4] ${ }^{8}, Q_{L}=3, Q_{d}=3, n=3, c_{2}=1$, $\omega=2 \pi \cdot 1.7 \cdot 10^{9} \mathrm{rad} / \mathrm{sec}$ and the transistor technology is 0.13 um CMOS (thick oxide) for which $V_{B D} \approx 2.5 \cdot 3.56 \mathrm{~V}^{9}$, $c_{1} \approx 4$ and $R_{\text {on }} C_{\text {out }} \approx 10^{-12}$; resulting in $m \approx 0.011$.

${ }^{7}$ Here $V_{B D}$ refers to gate-drain oxide breakdown voltage which is assumed to be lower than junction breakdown voltages. It is also assumed that $V_{D D_{\text {driver }}}$ is chosen as max. reliable gate-source oxide breakdown voltage.

${ }^{8}$ Value of $q$ is calculated as 1.2 from the circuit element values in [4] and $Q_{m}$ for $L_{m}$ and $L_{0}$ (bondwire) is assumed to be 30 .

${ }^{9}$ In [4], breakdown voltage is doubled by using a cascode topology. 
In [4], an optimization procedure based upon approximations and simulation results is given for Class-E PAs. The $P A E$ measurement results given in [4] $(67 \%$, for conventional ClassE PA) is close to theoretical value given in Fig. $3(\approx 71 \%)$. The difference can be attributed to the losses due to ground bonding and dc-feed inductance; which are not taken into account in the analytical design equations in this paper.

Fig. 3 shows the strong dependence of $P A E$ and $P_{\text {antenna }}$ both on $q$ and $\alpha$. It can be seen in Fig. 3 that the maximum $P_{\text {antenna }}$ for Class- $\mathrm{E}_{V V}(q=0.6, \alpha=2)$ is 1.65 times higher than the maximum $P_{\text {antenna }}$ for conventional Class-E PA ( $q=$ $1.2, \alpha=0)$. Besides, for $q=0.6$ Class- $_{V V}(\alpha=1)$ can have $\approx 80 \%$ more $P_{\text {antenna }}$ with only $3 \%$ less PAE than conventional Class-E PA $(\alpha=0)$. For the same $q=0.6$, Class-E $_{V V}(\alpha=2)$ can have $\approx 200 \%$ more $P_{\text {antenna }}$ with $\approx 16 \%$ less $P A E$ than the conventional Class-E PA $(\alpha=0)$.

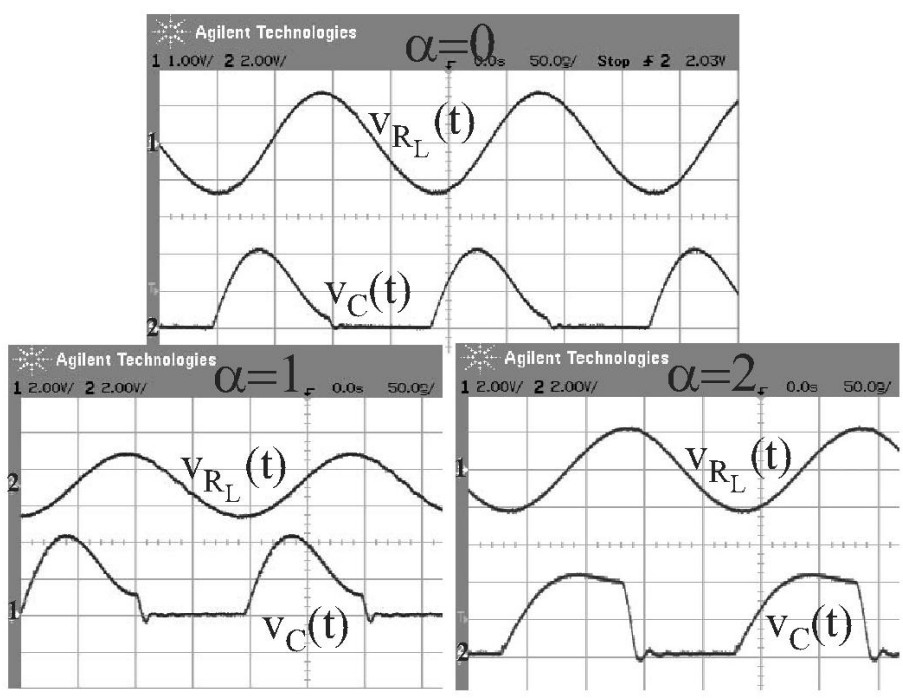

Fig. 4. Measurement Results for $\alpha=0,1$ and 2

\section{Proof of Concept Design}

We verified the given design equations in this paper by simulating the model in Fig.2b by transient and pss (periodic steady state) simulations in Spectre (Cadence). Very good agreement in the waveforms is observed between the simulations and the theory with a discrepancy of $\approx 3 \%$; attributed to the finite value of $Q_{L}=5$ used in the simulations.

Measurement results at low frequency $(5 \mathrm{MHz})$ are given in Table 2 and Fig. 4; which verify that Class- $\mathrm{E}_{V V}$ can have higher output power with reasonable efficiency in comparison to conventional Class-E. The three PAs in Table 2 are implemented on pcb by using discrete transistor (start499) with maximum allowed peak voltage $\left(v_{C} \approx 4.5 \mathrm{~V}\right)$, ceramic capacitors and air-core inductors. In the measurements, a 54642A Oscilloscope, 34401A DMV and a E3631A DC power supply are used. The switch (transistor) of the Class-E PA is directly driven with a square signal from an Agilent 33250A signal source. Due to lack of the RF source at low frequency $P_{\text {drive }}$ couldn't be measured but is calculated to be $\approx 3 \mathrm{~mW}$.
Table 2: Measurement Results and Design Details

\begin{tabular}{|c|c|c|c|}
\hline Design Details & Class-E & Class-E ${ }_{V V_{\alpha=1}}$ & Class-E $\mathbf{E}_{V V_{\alpha=2}}$ \\
\hline$\left(\mathrm{q}, \alpha, \mathrm{m}, Q_{L}, \mathrm{n}\right)$ & $(0,0,0.001,5,2.3)$ & $(0,1,0.001,5,2.3)$ & $(0, \mathbf{2}, 0.001,5,2.3)$ \\
\hline Frequency, $V_{D D}$ & $5 \mathrm{MHz}, 1.4 \mathrm{~V}$ & $5 \mathrm{MHz}, 1.6 \mathrm{~V}$ & $5 \mathrm{MHz}, 1.9 \mathrm{~V}$ \\
\hline$P_{O U T}, P_{D C}$ & $19.9,29.4 \mathrm{~mW}$ & $\mathbf{3 0 . 4}, 48.4 \mathrm{~mW}$ & $\mathbf{5 5 . 2}, 108.3 \mathrm{~mW}$ \\
\hline $\mathrm{R}, \eta$ & $22 \Omega, 67.7 \%$ & $22 \Omega, \mathbf{6 2 . 8 \%}$ & $22 \Omega, 51.0 \%$ \\
\hline$L_{m}, \mathrm{~L}, V_{\text {peak }}$ & $1.6,12 \mathrm{uH}, 4.2 \mathrm{~V}$ & $1.4,12 \mathrm{uH}, 4.2 \mathrm{~V}$ & $1.3,12 \mathrm{uH}, 4.2 \mathrm{~V}$ \\
\hline C, $C_{m}$ & $0.26,0.72 \mathrm{nF}$ & $0.42,0.72 \mathrm{nF}$ & $0.79,0.72 \mathrm{nF}$ \\
\hline
\end{tabular}

\section{CONCLUSION}

This paper shows the analytical design equations, and the time domain analytical solution for Class- $\mathrm{E}_{V V}$ PAs, including finite dc-feed inductance and switch input/output capacitance and on-resistance. In comparison to conventional Class-E PAs, Class- $\mathrm{E}_{V V}$ PAs can have lower peak switch voltage; which can be utilized to obtain high output power using lowvoltage transistor technologies (e.g. CMOS). This paper shows (theoretically and experimentally) that Class- $\mathrm{E}_{V V}$ PAs can have up to $\approx 200 \%$ more output power than conventional Class-E PAs under the same drive, load and reliable peak voltage conditions, with only a modest $P A E$ penalty.

\section{REFERENCES}

[1] T. Suetsugu, M.K. Kazimierczuk, "Analysis of Sub-Optimum Operation of Class E Amplifier" Circuits and Systems, Proceedings of the 46th IEEE International Midwest Symposium, vol. 3, pp: 1071- 1074, Dec. 2003.

[2] D.Y.C. Lie, et al "The limitations in applying analytic design equations for optimal class E RF power amplifiers design" VLSI Design, Automation and Test, IEEE 2005, pp:161 - 164

[3] F.H. Raab, "Idealized Operation of The class E Power Amplifier," IEEE Trans. Circuit. Syst., vol. 24. no. 12, Dec. 1977.

[4] Mazzanti, A,et al "Analysis of reliability and power efficiency in cascode class-E PAs," IEEE, JSSC, May 2006, vol.24, pp:1222 - 1229.

[5] M. Acar, A.J. Annema, B. Nauta "Generalized Design Equations for Class-E Power Amplifiers with Finite DC Feed Inductance" 36th European Microwave Conference, September 2006, pp. 13081311.

[6] M. Acar, A.J. Annema, B. Nauta "Generalized Analytical Design Equations for Variable Slope Class-E Power Amplifiers" 13th IEEE International Conference on Electronics, Circuits and Systems, December 2006.

[7] M. Acar, A.J. Annema, B. Nauta "Analytical Design Equations for ClassE Power Amplifiers with Finite DC Feed Inductance and Switch On Resistance" IEEE, International Symposium on Circuits and Systems, May 2007 (accepted).

\section{APPENDIX I}

$p$ and $\varphi$ are expressed in terms of $q, m$ and $\alpha$ as follows:

$$
\begin{aligned}
p= & \frac{\sqrt{\left(g_{1} h_{2}-g_{3} h_{3}\right)^{2}+\left(h_{1} g_{3}-h_{2} g_{2}\right)^{2}}}{-g_{1} h_{1}+g_{2} h_{3}}, \varphi=\arctan \left(h_{1} g_{3}-h_{2} g_{2}, g_{1} h_{3}-g_{3} h_{1}\right) \\
g_{1}= & \frac{-e^{a \pi}\left(A+b m q^{2}\right)+e^{b \pi}\left(A+a m q^{2}\right)}{B(-b+a)}-\frac{q \sin (q \pi)}{q^{2}-1}+\frac{m q^{2}}{B} \\
g_{2}= & -\frac{(\cos (q \pi)+1) q^{2}}{q^{2}-1}+\frac{m^{2} q^{2}(q-1)(q+1)}{B}+\frac{-e^{a \pi}\left(A b-m q^{2}\right)+e^{b \pi}\left(A a-m q^{2}\right)}{B(-b+a)} \\
g_{3}= & \frac{e^{b \pi}(-\alpha+(1-\alpha) m a)-e^{a \pi}(-\alpha+(1-\alpha) m b)}{-b+a}-\cos (q \pi)(1-\alpha) \\
h_{1}= & -\frac{m^{2} q^{2}}{B}+\frac{q^{2}(m \sin (q \pi) q-\cos (q \pi)-1)}{q^{2}-1}+m\left(\frac{a e^{a \pi}\left(A b-m q^{2}\right)}{B(-b+a)}-\frac{b e^{b \pi}\left(A a-m q^{2}\right)}{B(-b+a)}\right) \\
h_{2}= & (1-\alpha)(-\cos (q \pi)+m \sin (q \pi) q)+1+\frac{a e^{a \pi}(-\alpha+(1-\alpha) m b)}{-b+a}-\frac{b e^{b \pi}(-\alpha+(1-\alpha) m a)}{-b+a} \\
h_{3}= & \frac{m^{3} q^{2}(q-1)(q+1)}{B}-\frac{q(m \cos (q \pi) q+\sin (q \pi)+m q)}{q^{2}-1}+\frac{m a e^{a \pi}\left(A+b m q^{2}\right)}{B(-b+a)} \\
& -\frac{m b e^{b \pi}\left(A+m q^{2} a\right)}{B(-b+a)}, A=\left(q^{4}-q^{2}\right) m^{2} \text { and } B=1+m^{2} q^{4}-2 m^{2} q^{2}+m^{2}
\end{aligned}
$$

